\title{
RESEARCHES OF THE COMBINATORIAL MODELS FOR INNOVATIVE INFORMATION TECHNOLOGIES
}

\author{
Volodymyr Riznyk ${ }^{1)}{ }^{2)}$ \\ 1) Lviv Polytechnic National University, 12 S.Bandera Str., 79013, Lviv, Ukraine, rvv@polynet.lviv.ua, \\ http://iknit.lp.edu.ua/riznyk \\ ${ }^{2)}$ University of Technology and Life Sciences Bydgoszcz, 7 Kaliskiego Ale, 85-796, Poland, wriz@utp.edu.pl
}

\begin{abstract}
The paper presents a new mathematical principle of innovative techniques for improving the quality indices of engineering devices and systems with non-uniform structure (e.g. coding systems) with respect to transmission speed, positioning precision, resolving ability, and functionality, using novel design based on remarkable properties and structural perfection of one- and multidimensional models of the systems, namely the concept of Ideal Ring Bundles (IRB)s prospected from basic laws of the world-wide harmony. Research into the underlying mathematical principle provides an ability to reproduce the maximum number of combinatorial varieties in the systems with a limited number of elements and bonds. This approach make it possible to configure systems with optimal placement of structural elements in spatially or temporally distributed systems, using the appropriate mathematical apparatus of contemporary combinatorial theory.
\end{abstract}

Keywords: Combinatorial model, Information technology, Numerical model, Coding system, Transmission speed, Resolving ability, Multidimensional model, Vector data coded design, Ideal Ring Bundle, World-wide harmony.

\section{INTRODUCTION}

Problem of structural optimization in systems engineering relating to finding the best placement both elements and bonds of the system as well as improving its quality indices, is known, to be connected not only with number theory and combinatorial analysis but also with research of the fundamental laws of real world, such as symmetry laws [1], [2] and aurea section [2]. Research into underlying mathematical area involves investigation of novel techniques based on Combinatorial Mathematics, and Combinatorial Sequencing Theory, namely the concept of Ideal Ring Bundles [3], which can be used for finding optimal solutions for some problems in systems engineering and information technologies.

\section{NUMERICAL MODEL OF HIGH- PERFORMANCE SYSTEMS}

The ordered chain approach to the study of elements and events is known to be of widespread applicability when applied to the problem of finding the optimum ordered (non-redundant) arrangement of structural elements in a distributed technological or computing system. Let us regard an $n$-stage sequence of distinct positive integers $K_{n}=\left\{k_{1}, k_{2}\right.$, $\left.\ldots k_{n}\right\}$ as being cyclic, so that $k_{n}$ is followed by $k_{1}$.
We call this a ring sequence (Fig.1). A sum of consecutive terms in the ring sequence can have any of the $n$ terms as its starting point, and can be of any number of terms from 1 to $n-1$. In addition, there is the sum of all $n$ terms, which is the same independent of the starting point. Hence, the maximum number of distinct sums $S_{n}$ of consecutive terms of the ring sequence is given by:

$$
S_{n}=n(n-1)+1
$$

Here is graphical model of an $n$-stage ring sequence $K_{n}=\left\{k_{1}, k_{2}, \ldots k_{n}\right\}$ (Fig. 1$)$.

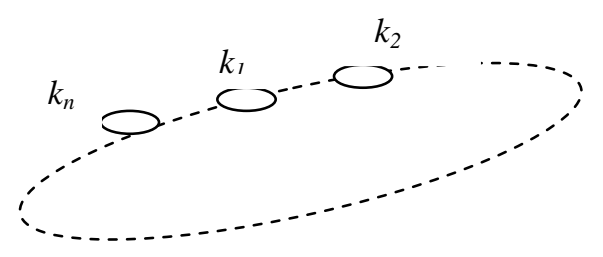

Fig.1 - Graphical model of an $\boldsymbol{n}$-stage ring sequence

$$
K_{n}=\left\{k_{1}, k_{2}, \ldots k_{n}\right\}
$$

If we require all terms in each sum to be consecutive elements of the sequence, for which the set of all $S_{n}$ circular sums consists of the numbers from 1 to $S_{n}=n(n-1)+1$ that is each number occurs exactly once is called Ideal Ring Bundle (IRB) with 
parameters $n, S_{n}$. Here is an example of an IRB with parameters $n=4, S_{n}=13$, namely $\{1,3,2,7\}$ (Fig.2).

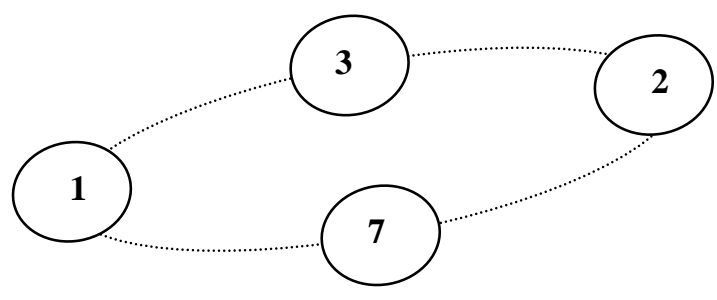

Fig. 2 - The Ideal Ring Bundle with $n=4, S_{n}=13$

To see this, we observe complete set of circular sums calculated from the IRB $\{1,3,2,7\}$ (Table 1).

Table 1. Circular sums of the IRB $\{1,3,2,7\}$

\begin{tabular}{|l|l|l|l|}
\hline $1=1$ & $2=2$ & $3=3$ & $4=1+3$ \\
\hline $5=3+2$ & $6=1+3+2$ & $7=7$ & $8=7+1$ \\
\hline $9=2+7$ & $10=2+7+1$ & $11=7+1+3$ & $12=3+2+7$ \\
\hline $13=1+3+2+7$ \\
\hline
\end{tabular}

Note that if we allow summing over more than one complete revolution around the ring, we can obtain all positive integers as such sums. Thus:

$$
\begin{aligned}
& 14=1+3+2+7+1,15=2+7+1+3+2, \\
& 16=3+2+7+1+3, \text { etc. }
\end{aligned}
$$

One-dimensional (1-D) Ideal Ring Bundle is an ordered numerical construction with $\mathrm{n}$ distinct integers, which form perfect partitions of finite interval $[1, \mathrm{~s}]$ of integers. The sums of connected sub-sequences of 1-D IRB enumerate the set integers $[1, \mathrm{~s}]$ exactly R-times [3].

The favorable qualities of numerical models provide many opportunities to apply them to advanced information technology and numerous branches of science.

\section{IRB CODING SYSTEMS}

Concept of the Ideal Ring Bundles can be used for finding optimal solutions for some problems of information computational processes. For example, Ideal Ring Bundles make it possible to configure optimum coding system, based on so-called IRB Monolithic Binary Code. This code forms binary code combinations, which all symbols " 1 " as well as symbols " 0 " are arranged together [4]. The IRBs provide an ability to reproduce the maximum number of the "monolithic" code combinations (all symbols " 1 " as well as symbols " 0 " are arranged together) in the coding system with a limited number of code word digits. First of all we can see computational processes, based on the IRB Monolithic Binary Code.

Here is an example of one-dimensional Monolithic Binary Code based on the IRB $\{1,3,2,7\}$ with parameters $n=4, S=13$ :
Table 2. One-dimensional Monolithic Binary Code based on the IRB $\{7,2,3,1\}$

\begin{tabular}{|c|c|}
\hline Number & Code combination \\
\hline 0 & 0000 \\
\hline 1 & 0001 \\
\hline 2 & 0101 \\
\hline 3 & 0010 \\
\hline 4 & 0011 \\
\hline 5 & 0110 \\
\hline 6 & 0111 \\
\hline 7 & 1000 \\
\hline 8 & 1001 \\
\hline 9 & 1100 \\
\hline 10 & 1101 \\
\hline 11 & 1011 \\
\hline 12 & 1110 \\
\hline 13 & 1111 \\
\hline
\end{tabular}

Table 2 contains the complete set of binary code combinations for coding the numbers $0,1, \ldots 13$.

Underlying combinatorial constructions can be represented as mathematical model of optimum coding system, based on the IRBs conception.

\section{GENERALIZED IDEAL RING BUNDLES}

Next, we consider a more general type of IRB, where the $S_{n}$ circular sums of consecutive terms give us each integer value from 1 to $N$, for some integer $N$, exactly $R$ times, as well as the value $N-1$ (the sum of all $n$ terms) exactly once. Here we see that:

$$
N=n(n-1) / R
$$

Here is a graph of the IRB with $n=4, R=2$ and $N=6$, where $\left.k_{1}=1, k_{2},=1, k_{3}=2, k_{4}=3\right\}$ (Fig. 3 ).

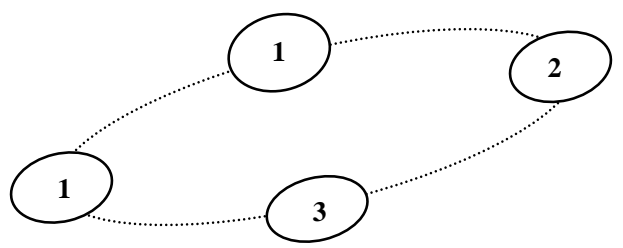

Fig. 3 - Graph of the IRB with $n=4, N=6, R=2$

Sums of consecutive terms for the IRB $\{1,1,2$, 3 ) are:

$\begin{array}{llll}\text { 1: } & 1, & \text { and } & 1, \\ \text { 2: } & 1+1, & \text { and } & 2, \\ \text { 3: } & 1+2, & \text { and } & 3, \\ \text { 4: } & 1+1+2 & \text { and } & 3+1, \\ \text { 5: } & 2+3 & \text { and } & 3+1+1, \\ \text { 6: } & 1+2+3 & \text { and } & 2+3+1\end{array}$

Using equation (2) easy to calculate sums of all $n$ terms for double $(\mathrm{R}=2) \mathrm{IRB}$, namely: $2,4,7,11$, etc. We say this numerical set is generative double-IRBs 
row.

\section{SEGMENTATION OF IDEAL RING BUNDLES}

Let us regard of the IRB $\{1,3,2,7\}$ depicted above (Fig.2) and the IRB $\{1,1,2,3\}$ (Fig.3) as graphic vision of an image segmentation of the IRBs (Fig.4).
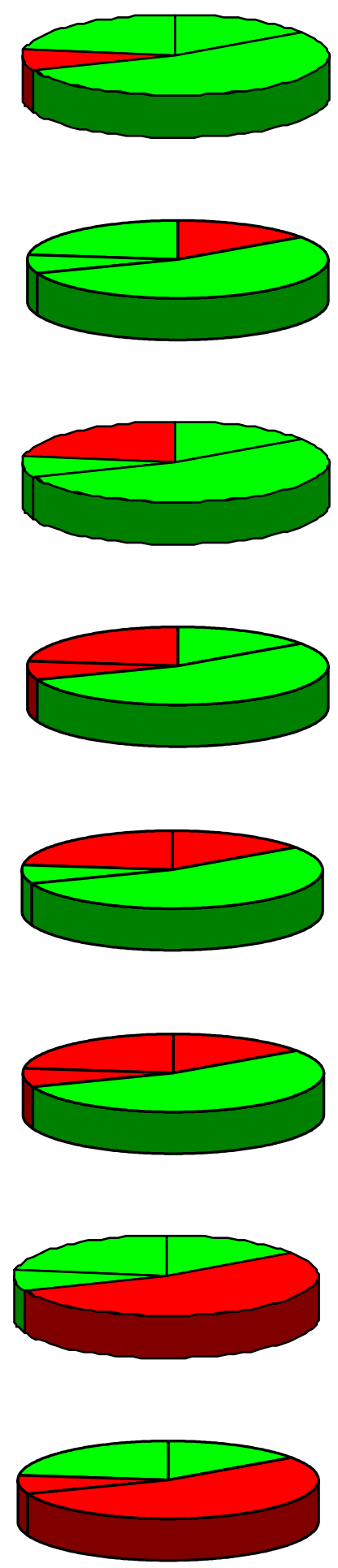
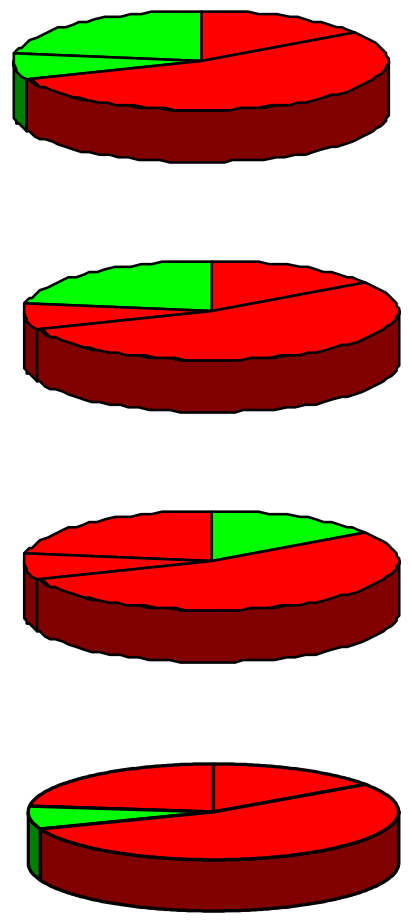

Fig. 4 - Graphic segmentation of the IRB $\{1,3,2,7\}$

Regarding picture (Fig.4), easy to see exhaustive set of harmonious two-body relationships (here is red and green) from 1:12 to $1: 12$ obtained exactly once $(R=1)$ each of them using the IRB $\{1,3,2,7\}$ image segmentation.

So, 13 is a lucky number, because it allows on the perfect partition $13=1+3+2+7$ and provides an ability to reproduce the maximum number of harmonious two-body relationships. The fact makes it possible to develop of new approach in research of the discrete structures from epistemological point of view or "nature and nurture" [2].

Now, let us regard a graphic segmentation of the Ideal Ring Bundle $\{1,1,2,3\}$. Here is exhaustive set of harmonious two-body relationships from 1:6 to $6: 1$ obtained exactly twice $(R=2)$ each of them over the IRB $\{1,1,2,3\}$ (Fig. 5).

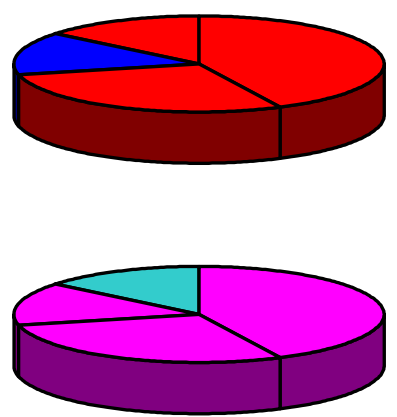



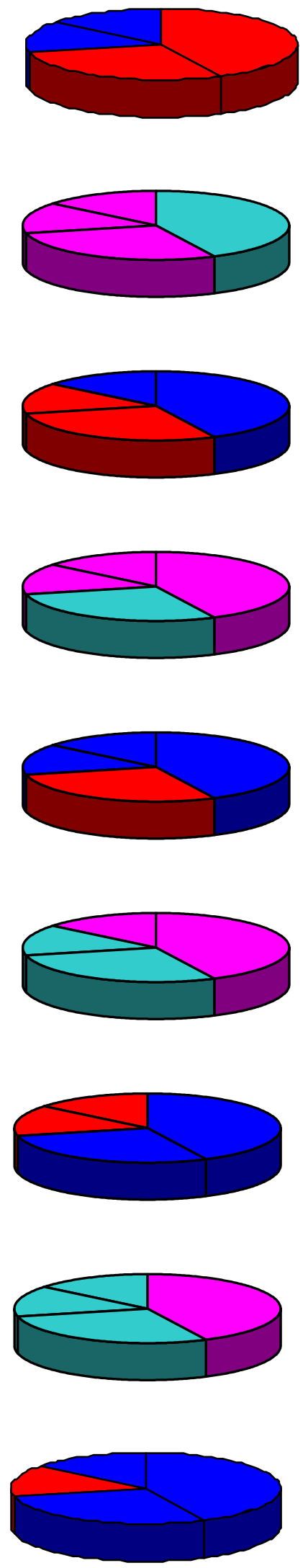

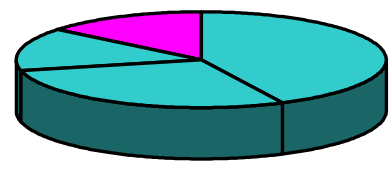

Fig. 5 - Graphic segmentation of the IRB $\{1,1,2,3\}$

Observing graphic segmentation of the $\operatorname{IRB}\{1,1$, 2, 3 \} (Fig.5), we see the exhaustive set of harmonious two-body relationships (here is blue and red, and sky-blue and lilac) from 1:6 to $1: 6$ obtained exactly twice $(R=2)$ each of them. So, 7 is lucky number, because it provides an ability to reproduce the exhaustive set of harmonious two-body relationships.

\section{PERFECT NON-SYMMETRY AND SYMMETRY OF THE IDEAL RING BUNDLES}

We can see, that neither IRB $\{1,3,2,7\}$ nor IRB $\{1,1,2,3\}$ are symmetric structures. Now let us consider a central symmetric figure of order 7 (Fig.3). Easy to see, that it is a chart of the IRB $\{1$, $1,1,1,1,1,1\}$ with $n=7, S_{n}=7$, and $R=7$. This chart consists of two IRBs, which penetrate with each other, namely the IRB $\{1,1,2,3\}$ (black bubbles) and the IRB $\{1,2,4\}$ (red bubbles).

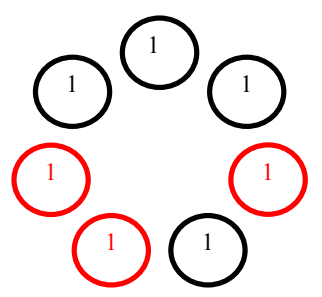

Fig. 6 - Bubble chart of the symmetric IRB $\{1,1,1,1$, $1,1,1\}$ joined two non-symmetric IRBs $\{1,1,2,3\}$ and $\{1,2,4\}$

There are, it is known, numerous numbers of the underlying perfect triads of IRBs, which form socalled universal numerical information field [5]. It is, essentially, a fundamental property of the spacetime. The mutual connection between symmetry and non-symmetry of the IRBs provides a better understanding of physics of space-time, and role of geometric structure in the behaviour of natural and man-made objects.

\section{IDEAL RING BUNDLE AS SYMMETRIC OBJECT}

Next, we regard the $n$-stage ring sequence $K_{2 D}=\left\{\left(k_{11}, k_{12}\right),\left(k_{21}, k_{22}\right) \ldots\left(k_{n 1}, k_{n 2}\right)\right\}$, where we require 
all terms in each circular vector-sum to be consecutive 2-stage sequences as elements of the sequence. A circular vector-sum of consecutive terms in the ring sequence can have any of the $n$ terms as its starting point, and can be of any length from 1 to $n-1$. An $n$-stage ring sequence $K_{2 D}$, for which the set of all circular vector-sum forms twodimensional grid, where each node of the grid occurs exactly $R$-times, is named a two-dimensional Ideal Ring Bundle (2-D IRB).

Here is two-dimensional $n$-stage ring sequence IRB with four $(n=4)$ terms in the ring topology, where $k_{1}=(0,2), k_{2}=(1,0), k_{3}=(1,1), k_{4}=(2,2)$, which grid chart is depicted below (Fig.7).

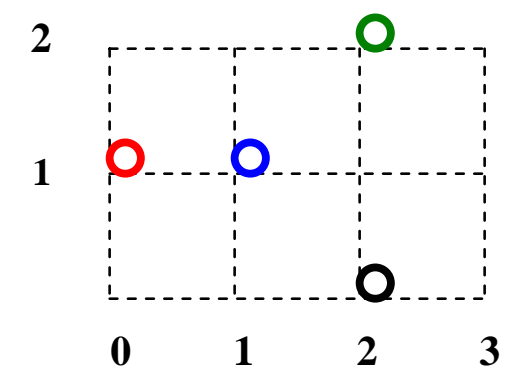

\section{Fig. 7. Grid chart of two-dimensional Ideal Ring Bundle (2-D IRB) of four $(n=4)$ vectors $\{(0,2),(1,0),(1,1),(2,2)\}$}

We can calculate easy the all circular twodimensional vector-sums, taking modulo $m_{1}=3$ for the first component of vector-sum and modulo $m_{2}=4$ for the second its component:

$$
\begin{aligned}
& (0,0) \equiv(2,2)+(0,2)+(1,0), \\
& (0,1) \equiv(1,1)+(2,2)+(0,2), \\
& (0,2)=(0,2), \\
& (0,3) \equiv(1,1)+(2,2), \\
& (1,0)=(1,0), \\
& (1,1)=(1,1), \\
& (1,2) \equiv(0,2)+(1,0), \\
& (1,3) \equiv(1,0)+(1,1)+(2,2), \\
& (2,0) \equiv(2,2)+(0,2), \\
& (2,1) \equiv(1,0)+(1,1), \\
& (2,2)=(2,2), \\
& (2,3) \equiv(0,2)+(1,0)+(1,1)
\end{aligned}
$$

So long as the vectors $(0,2),(1,0),(1,1),(2,2)$ of the ring sequence themselves are circular vectorsums too, the circular vector-sums set configure the $3 \times 4$ matrix as follows:
$(0,0)$
$(0,1)$
$(0,2)$
$(0,3)$
$(1,0)$
$(1,1) \quad(1,2)$
$(1,3)$
$(2,0)$
$(2,1)$
$(2,2)$
$(2,3)$

The result of the calculation forms the $3 \times 4$ grid which exhausts the circular $2-\mathrm{D}$ vector-sums and each of its meets exactly once $(R=1)$. So, the ring sequence of the 2 -D vectors $\{(0,2),(1,0),(1,1),(2$, $2)\}$ is two-dimensional Ideal Ring Bundle (2-D IRB) with $n=4, R=1$, and $m_{1}=3, m_{2}=4$.

It is easy to see, that $2-\mathrm{D} \operatorname{IRB}\{(0,2),(1,0),(1$, $1),(2,2)\}$ has the mirror symmetry, as well as differences of coordinates for all pairs of elements (bubbles on the grid chart) form a set of nonidentical positions of 2-D vectors. So, the $2-\mathrm{D}$ IRB is both symmetric and non-redundant structure at the same time. There are lots of symmetric and nonredundant 2-D IRBs. It is known, we can configure numerous numbers of one- and multidimensional IRBs [4].

As we see a whole can be partitioned into two parts using corresponding couple of rays, where one part of two partners of an arbitrary polarity is designed with the sign " + " and the other part of this polarity is designed with the sign "--", so in the following we shall always speak of the IRB polarity.

Perfect Distribution Law (PDL), based on the remarkable properties and structural perfection of one- and multidimensional IRBs are as follows:

A whole (space-time) can be partitioned perfectly, namely the sums of connected parts of the whole enumerate the set of harmony rising its parts exactly $R$-times with the smallest possible number of intersections.

The main characteristics of the PDL [5]:

1. A whole can be partitioned perfectly by infinitely great number of ways.

2. The minimal share of a whole in perfect partitioning can be anyone small.

3. The perfect partitioning can be useful to objects of anyone vector space dimensionality.

\section{PERFECT VECTOR DATA CODING DESIGN}

The development of new direction in fundamental and applied research in information technologies is connected with vector data coding design, based on the concept of Ideal Ring Bundles. For example, underlying combinatorial construction can be represented as mathematical model of optimum coding system, based on so-called "Monolithic Binary Code" (MBC). This code forms binary code combinations which all symbols " 1 " as well as "0" are arranged together. For example, twodimensional vector data MBC formed on the 2-D $\operatorname{IRB}\{(0,2),(1,0),(1,1),(2,2)\}$, is given in the Table 3 . 
Table 3. 2-D MBC based on the irb \{(0,2), (1,0), $(1,1)$, $(2,2)\}$

\begin{tabular}{|c|c|c|c|c|c|}
\hline Vector & Code & Vector & Code & Vector & Code \\
\hline$(0,0)$ & 1101 & $(1,0)$ & 0100 & $(2,0)$ & 1001 \\
\hline$(0,1)$ & 1011 & $(1,1)$ & 0010 & $(2,1)$ & 0110 \\
\hline$(0,2)$ & 1000 & $(1,2)$ & 1100 & $(2,2)$ & 0001 \\
\hline$(0,3)$ & 0011 & $(1,3)$ & 0111 & $(2,3)$ & 1110 \\
\hline
\end{tabular}

Table 3 contains set of binary code combinations for coding of all 2 -D vectors on the $3 \times 4$ - matrix from $(0,0)$ (code combination 1101) to $(2,3)$ (code combination 1110), where each of them has been coded in circular 2-D MBC. The remarkable properties of the $\mathrm{MBC}$ provide its some advantages over the rest codes, involving simplicity of error detecting and correcting as well as high-speed operation.

\section{CONCLUSION}

The Perfect Distribution Law (PDL) discovers, essentially, a new scientific conception for development fundamental and applied research in information technologies, based on the idea of "perfect" combinatorial constructions- Ideal Ring Bundles (IRB)s, and the development of new directions in fundamental and applied research in area of information technologies, for improving such quality indices as reliability, precision, speed, resolving ability, and functionality. Structural perfection and remarkable properties of one- and multidimensional IRBs provide an ability to reproduce the maximum number of combinatorial varieties in the systems with a limited number of elements and bonds. It was IRBs provide many opportunities to apply them to advanced information technologies. The innovative methodologies based on combinatorial techniques of the Ideal Ring Bundles theory can be used for finding optimal solutions for wide classes of technological problems. Perspective information technologies and coded design prospected from the PDL are for example high performance vector data coding and selfchecking code, vector computing machinery and low side-lobe antenna design [4]. The mutual connection between symmetry and non-symmetry of the IRBs provides a better understanding of physics of spacetime, and role of geometric structure in the behaviour of natural and man-made objects. A perfection, beauty and harmony exists not only in the abstract models but in the real world also.

\section{REFERENCES}

[1] P.Wigner, Symmetries and Bloomington-London: Indiana University
Press, 1970.

[2] H. Weyl, Symmetry. Princeton, NJ: Princeton University Press, 1952.

[3] V. Riznyk. Combinatorial Sequencing Theory and its Applications, Lehrstuhl für Informatik V, Universität Mannheim (report), Germany 10 October 1997.

[4] S.Golomb, P.Osmera and V.Riznyk. Combinatorial Sequencing Theory for optimization of signal data vectors converting and signal processing. Proceedings of the Workshop on Design Methodologies for Signal Processing, Zakopane, Poland 29-30 August 1996, pp.43-44.

[5] V.Riznyk.Perspective information technologies prospected from basic laws of the world-wide harmony. Proceedings of the Third International Conference on Computer Science and Information Technologies (CSIT'2008), Lviv, Ukraine 25-27 September 2008, pp.8084.

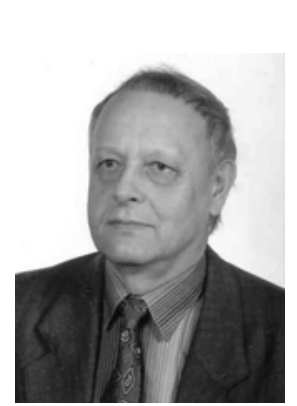

Volodymyr V. Riznyk born in Polis'ke, Kyiv Region, Ukraine, 1940. Education: Diploma, Power Engineering, 1962; Diploma, Radio Engineering, 1967; PhD Degree, Cybernetics and Theory of Information, Physical and Mechanical Institute, Academy of Sciences, Ukr. SSR, 1980; D Sc, Mathematical Modeling and Mathematical Techniques for Scientific Researches, Vinnytsia State Technical University, 1994. Full professor in Lviv Polytechnic National University, 1995, University of Technology and Life Sciences Bydgoszcz, Poland, 1996.

Scientific interests: researches and applications of combinatorial models and techniques to problems of information technologies. 